\title{
Present moment, past, and future: mental kaleidoscope
}

\section{Andrew A. Fingelkurts * and Alexander A. Fingelkurts}

BM-Science - Brain and Mind Technologies Research Centre, Espoo, Finland

*Correspondence: andrew.fingelkurts@bm-science.com

Edited by:

James M. Broadway, University of California Santa Barbara, USA

Reviewed by:

Valdas Noreika, Medical Research Council, UK

Keywords: subjective present moment, operation, subjective time flow, brain operational architectonics, EEG

It is the every person's daily phenomenal experience that conscious states represent their contents as occurring now. Following Droege (2009) we could state that consciousness has a peculiar affinity for presence. Some researchers even argue that conscious awareness necessarily demands that mental content is somehow held "frozen" within a discrete progressive present moment (James, 1890; Lynds, 2003). Thus, phenomenal content seems to be minimally conscious if it is integrated into a single and coherent model of reality during a "virtual window" of presence (Metzinger, 2003; see also Brown, 1998; Varela, 1999; Smythies, 2003).

In order to explain such features of consciousness as phenomenal unity and continuity within the current present along with a succession of discrete thoughts that give rise to feeling of the past and future, a reference to mechanisms outside the phenomenal realm is necessary (Revonsuo, 2003). Thus, the question of what could be the neurophysiological mechanisms responsible for these experiences should be addressed.

In this Opinion Article we shall build our argument based on the biological realism approach to consciousness proposed by Revonsuo (2006). According to this approach, subjective consciousness is a real phenomenon that is tightly anchored to a biological reality within the human brain. Broadly speaking, the human brain is the specific physical "location," where the subjective mental reality and the objective neurobiological reality are intimately connected along a unified metastable continuum (Fingelkurts et al., 2009, 2013).

We have argued previously (Fingelkurts et al., 2010) that phenomenal consciousness refers to a higher level of organization in the brain and captures all immediate and undeniable (from the first-person perspective) phenomena of subjective experiences (hearing, seeing, touching, feeling, embodiment, moving, and thinking) that present to any person right now (subjective present) and right here (subjective space). By this definition even remembering the past images and planning the future events can't be performed other than in the present moment and in relation to current state of affairs (see also Lynds, 2003; Droege, 2009). This is so because someone possesses phenomenal consciousness if there is any type of subjective experiences that is currently present for him/her (Fingelkurts et al., 2010).

In this context what is presented as now is not simply whatever sensory or other representations occur in the brain at any given moment but rather the spatialtemporal hierarchy of selected and nested metastable states of neuronal assemblies that serve in real time as a basis for the subjective experiences of the "present moment." Among many theories, the Operational Architectonics (OA) theory of brain and mind functioning (Fingelkurts and Fingelkurts, 2001, 2008; Fingelkurts et al., 2010, 2013) explicitly utilizes the hierarchy of nested metastable states of neuronal assemblies. In short, OA theory is centered on the notion of operation. Operation is broadly defined as the process or state of being in effect and it has a beginning and an end (Collins Essential English Dictionary, 2006). In fact, everything which can be represented by a process is an operation. The notion of operation plays a central role in bridging the brain-mind gap and makes it possible to identify what at the same time belongs to the mental level and to the neurophysiological level of brain activity organization, and acts as a mediator between the two (Fingelkurts and Fingelkurts, 2001, 2008; Benedetti et al., 2010). Understanding of the operation as a process and considering its combinatorial nature, seems especially well suited for describing and studying the mechanisms of how information about the objective physical entities of the external world can be integrated within the present moment in the internal subjective domain by means of entities of distributed neuronal assemblies (Fingelkurts et al., 2010, 2013). In line with this conceptualization, simple cognitive operations that present some partial aspect of the whole object/scene/concept are presented in the brain by local 3D-fields produced by discrete and transient neuronal assemblies, which can be recorded by an electroencephalogram (EEG) (Figures 1A,B). More complex operations that constitute the whole object or scene are brought into existence by joint (synchronized) simple operations in the form of coupled 3D-fields - so called operational modules (OMs) of varied complexity (Fingelkurts and Fingelkurts, in press). Further synchronization of several OMs (complex field spatial-temporal patterns; Figure 1A) forms even more coarse scales of nested functional hierarchy (Feinberg, 2000) that is now able to present and hold highly complex sensorial inputs as coherent perceptions of the world, create internal complex images and form conscious decisions (Fingelkurts et al., 2010, 2013). The recombination of neuronal assemblies and their operational modules into new configurations gives rise to a nearly inexhaustible source of presenting different qualities, patterns, objects, scenes, concepts and decisions. 


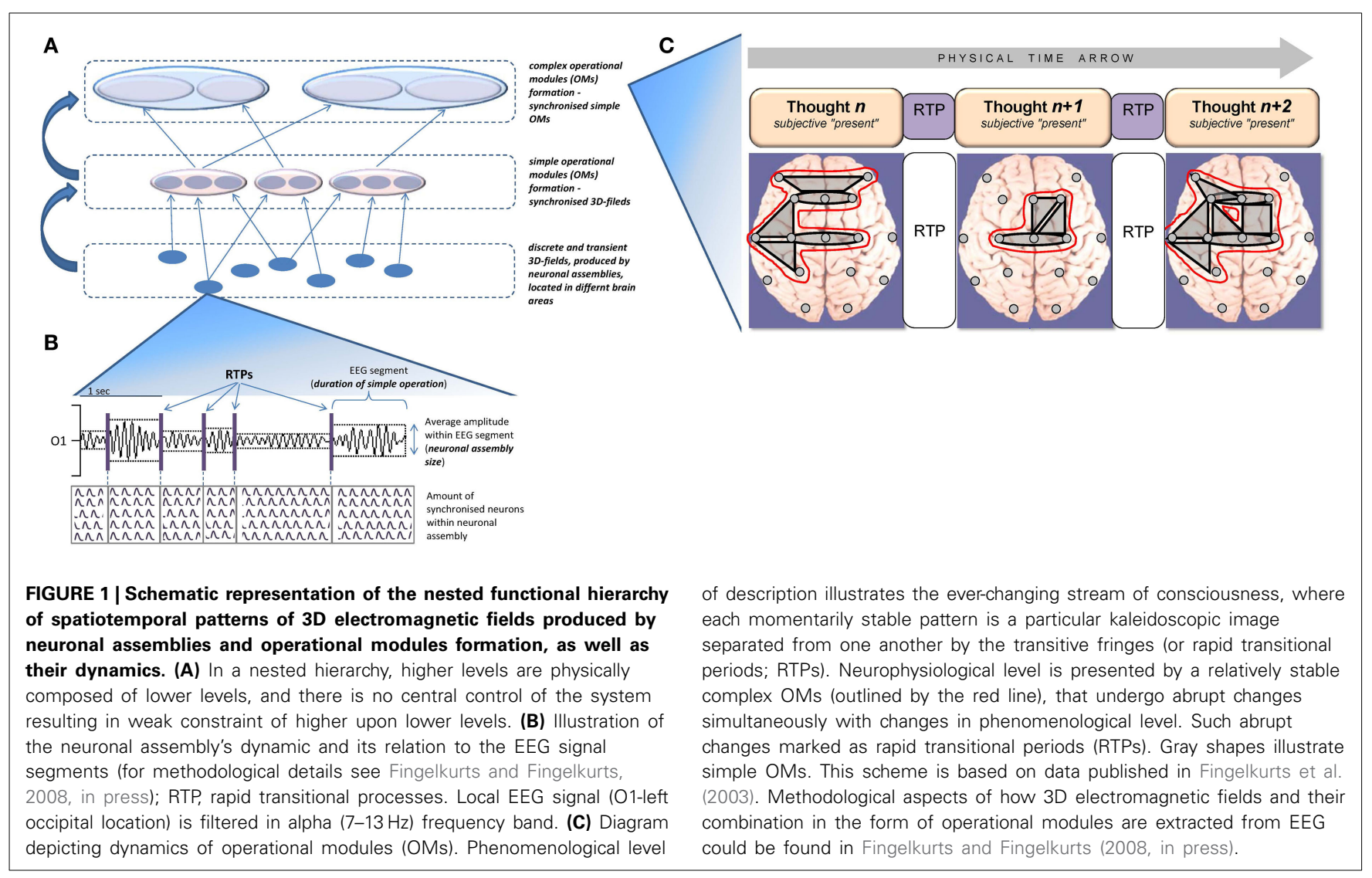

In the following we will discuss how the OA framework could implement the subjective present and some other temporal phenomena. We argue that at the phenomenological level, the lasting OM would be experienced as the "phenomenal present" of consciousness (Figure 1C). This hypothesis remains to be proven experimentally, however some empirical evidence already exists. For example, the mean duration of OMs (derived from an EEG with a frequency band of $0.3-$ $30 \mathrm{~Hz}$ ) usually varies from $80-100 \mathrm{~ms}$ for large OMs spanning the cortex to $30 \mathrm{~s}$ for small local OMs. These accounts, including duration variation, are consistent with known estimates for the frame of a specious present, which varies from $\sim 100 \mathrm{~ms}$ to several seconds depending on circumstances (Pöppel, 1988).

However, if the brain could implement only a complex but static OM, then such a brain would only experience the presence of one unified world frozen into an internal now (Metzinger, 2003). Neither the complex texture of subjective time flow, nor true perspectivalness that goes along with a first-person point of view would exist in such situation (Fingelkurts et al., 2010). Therefore, a dynamic succession of phenomenal moments that are integrated into the flow of subjective time is needed. Indeed, as it is evident from the first-person perspective, the actualization of full-fledged phenomenal objects, images or scenes is realized on a "one-at-a-time" basis, moving serially from one phenomenal pattern within a specious present to another (Revonsuo, 2006). This process gives rise to a stream of consciousness that is best conceptualized in the James' metaphor of a kaleidoscope (James, 1890). Using this metaphor James illustrates the ever-changing stream of thoughts like a rotating kaleidoscope where each momentarily stable pattern constructed from multiple pieces (local fields in our interpretation) is a speciously presented thought (OM in our interpretation). Thus, the succession of phenomenal images or thoughts is neurophysiologically presented by the succession of discrete and relatively stable OMs, which are separated by rapid transitive processes (RTP), i.e., abrupt changes of OMs (Figure 1C). As it has been shown experimentally, at the critical point of transition in mental states, the $\mathrm{OM}$ undergoes a profound reconfiguration which is expressed through the following process (Fingelkurts et al., 2000, 2003; Fingelkurts and Fingelkurts, in press): The OM, which is comprised from a set of local bioelectrical fields produced by transient neuronal assemblies across several brain areas, rapidly loses functional couplings and establishes new couplings within another set of local bioelectrical fields, thus demarcating a new $\mathrm{OM}$ in the volumetric operational spacetime continuum of the brain (Figure 1C).

Thus, the presented model for OM mediated succession of phenomenal images or thoughts is one way of understanding how subjective time flow is mentally (re)constructed beyond the phenomenal horizons of "presence." Subjective time flow is not actually experienced or "perceived," rather it emerges as the product of cognitive higher-order processes operating on the OMs (Fingelkurts et al., 2010). Such higher-order processes 
are also expressed in the form of complex OMs, that not only superceedes lower-level OMs, but also execute memory consolidation and retrieval operations (Fingelkurts et al., 2003). Given such a mechanism, the variation in subjectively experienced speed of time could be also explained. When the OMs' average duration decreases, there are many more OMs managing to sequence each other within a given time unit. We suggest that this overflow of OMs would be commonly experienced as an acceleration of the subjective time. Conversely, if the average duration of OMs was to increase, then the subjective experience of time would slow down. Below, we review some experimental evidence in support of our theorizing.

It is well known that certain psychoactive agents create subjective time distortions when administered. For example, opioids can be used to prolong the subjectively perceived duration of thought (Galski et al., 2000). In agreement with OA framework, it has been shown that opioids do indeed increase the duration of the lifespan of neuronal assemblies (indexed by EEG quasi-stationary segments) and limit the synchronization between their operations, thus reducing the possible number of OMs while increasing their life-span (Fingelkurts et al., 2006).

Another important model, where subjective experience could be easily manipulated is hypnosis. In a neutral hypnotic state the subject experiences an altered background state of consciousness different from the normal baseline state of consciousness without the need of suggestion (Kallio and Revonsuo, 2003). This subjective state is characterized by "emptiness" or "absorption" brought about by dissociations in the cognitive modules that are temporarily incapable of normal communication with each other (Gruzelier, 2000). Additionally, it has been shown that the subjective sensation for the passage of time is stretched during hypnosis, because internal events are subjectively slowed (Von Kirchenheim and Persinger, 1991; Naish, 2001). Adhering to the tenets of OA framework, these subjective experiences should be reflected in the operational architectonics of the electromagnetic brain field. It was indeed shown that the functional life-span of neuronal assemblies (indexed by the EEG quasi-stationary segments) was significantly longer during hypnosis when compared with the normal/baseline conscious condition (Fingelkurts et al., 2007). It was further found that the number and strength of synchronized operations among different neuronal assemblies were significantly lower during hypnosis than during the baseline, thus limiting the possibility for any OMs to emerge. As a result they were absent (Fingelkurts et al., 2007). Since OMs represent the formation of integrated conscious experiences, their absence may explain such unusual subjective experiences during hypnosis as amnesia, timelessness, detachment from the self, a "willingness" to accept distortions of logic or reality, and the lack of initiative or willful movement (Dietrich, 2003).

Dreaming is a special case where the phenomenal world is realized in the brain in its "pure form," because it is nearly completely isolated from the external physical world and the rest of the body. Dreams can appear in REM as well as in the nonREM sleep (Nir and Tononi, 2010). However, the nature of dreams in REM and nonREM sleep is different: during REM the dreams are complex, organized, temporally evolving, multimodal, and often bizarre (Hobson et al., 2000), while in nonREM the dreams are characterized by simple, static or isolated image(s) or though(s), usually of one modality (Noreika et al., 2009). The OA prediction is that nonREM dreams should be accompanied by shortlived small neuronal assemblies and longlived large neuronal assemblies, and by the significant increase of operational synchrony (poor set of OMs) among different neuronal assemblies in order to subjectively present static images or thoughts. In a pilot nonREM sleep study (Fingelkurts and Fingelkurts, in press) we found that nonREM dreams were indeed accompanied by the small short-lived and large long-lived neuronal assemblies, as well as significant operational synchrony increase in the OA organization of the brain. Future research should establish the OA data for REM sleep dreams.

This brief review of results supports the suggested neurophysiological mechanism (within the operational architectonics of the human brain field) responsible for the experiences of the "present moment," past, and future.

\section{ACKNOWLEDGMENTS}

The authors would like to thank Dmitry Skarin for English editing.

\section{REFERENCES}

Benedetti, G., Marchetti, G., Fingelkurts, A. A. and Fingelkurts, A. A. (2010). Mind operational semantics and brain operational architectonics: a putative correspondence. Open Neuroimag. J. 4, 53-69. doi: 10.2174/1874440001004020053

Brown, J. W. (1998). Fundamentals of process neuropsychology. Brain Cogn. 38, 234-245. doi: 10.1006/brcg.1998.1033

Collins Essential English Dictionary. (2006). Collins Essential English Dictionary, 2nd Edn. Glasgow, UK: Harper Collins Publishers.

Dietrich, A. (2003). Functional neuroanatomy of altered states of consciousness: the transient hypofrontality hypothesis. Conscious. Cogn. 12, 231-256. doi: 10.1016/S1053-8100(02)00046-6

Droege, P. (2009). Now or never: how consciousness represents time. Conscious. Cogn. 18, 78-90. doi: 10.1016/j.concog.2008.10.006

Feinberg, T. E. (2000). The nested hierarchy of consciousness: a neurobiological solution to the problem of mental unity. Neurocase 6, 75-81. doi: 10.1080/13554790008402762

Fingelkurts, A. A1., and Fingelkurts, A. A. (2001). Operational Architectonics of the human brain biopotential field: towards solving the mindbrain problem. Brain Mind 2, 261-296. doi: 10.1023/A:1014427822738

Fingelkurts, A. A1., and Fingelkurts, A. A. (2008). Brain-mind operational architectonics imaging. technical and methodological aspects. Open Neuroimag. J. 2, 73-93. doi: 10.2174/1874440000 802010073

Fingelkurts, A. A1., and Fingelkurts, A. A. (in press). "Operational architectonics methodology for EEG analysis: theory and results," in Modern Electroencephalographic Assesment Techniques: Theory and Applications. Neuromethods Book Series, ed. V. Sakkalis (New York, NY: Springer). doi: 10.1007/7657_2013_60

Fingelkurts, A. A1., Fingelkurts, A. A., Borisov, S. V., Ivashko, R. M., and Kaplan, A. Y. (2000). Spatial structures of human multichannel EEG quasistationary segments during memory task. Vestn. Moskovsk. Univer. 3, 3-10.

Fingelkurts, A. A1., Fingelkurts, A. A., Kallio, S., and Revonsuo, A. (2007). Cortex functional connectivity as a neurophysiological correlate of hypnosis: an EEG case study. Neuropsychologia 45, 1452-1462. doi: 10.1016/j.neuropsychologia.2006. 11.018

Fingelkurts, A. A1., Fingelkurts, A. A., Kivisaari, R., Autti, T., Borisov, S., Puuskari, V., et al. (2006). Increased local and decreased remote functional connectivity at EEG alpha and beta frequency bands in opioid-dependent patients. Psychopharmacology 188, 42-52. doi: 10.1007/ s00213-006-0474-4

Fingelkurts, A. A1., Fingelkurts, A. A., Krause, C. M., Kaplan, A. Y., Borisov, S. V., and Sams, 
M. (2003). Structural (operational) synchrony of EEG alpha activity during an auditory memory task. Neuroimage 20, 529-542. doi: 10.1016/S10538119(03)00305-7

Fingelkurts, A. A1., Fingelkurts, A. A., and Neves, C. F. H. (2009). Phenomenological architecture of a mind and operational architectonics of the brain: the unified metastable continuum. J. New Math. Nat. Comput. 5, 221-244. doi: 10.1142/S179300570 9001258

Fingelkurts, A. A1., Fingelkurts, A. A., and Neves, C. F. H. (2010). Natural world physical, brain operational, and mind phenomenal space-time. Phys. Life Rev. 7, 195-249. doi: 10.1016/j.plrev.2010.04.001

Fingelkurts, A. A1., Fingelkurts, A. A., and Neves, C. F. H. (2013). Consciousness as a phenomenon in the operational architectonics of brain organization: criticality and self-organization considerations. Chaos Solit. Fract. 55, 13-31. doi: 10.1016/j.chaos.2013.02.007

Galski, T., Williams, J. B., and Ehle, H. T. (2000). Effects of opioids on driving ability. J. Pain Symptom. Manage. 19, 200-208. doi: 10.1016/ S0885-3924(99)00158-X

Gruzelier, J. H. (2000). Redefining hypnosis: theory, methods and integration. Contemp. Hypn. 17, 51-70. doi: 10.1002/ch.193

Hobson, J. A., Pace-Schott, E. F., and Stickgold, R. (2000). Dream science 2000: a response to commentaries on dreaming and the brain. Behav. Brain Sci. 23, 1019-1035. doi: 10.1017/S0140525X00954025
James, W. (1890). The Principles of Psychology, Vol. 1. New York, NY: Dover. doi: 10.1037/10538-000

Kallio, S., and Revonsuo, A. (2003). Hypnotic phenomena and altered states of consciousness: a multilevel framework of description and explanation. Contemp. Hypn. 20, 111-164. doi: 10.1002/ ch. 273

Lynds, P. (2003). Subjective perception of time and a progressive present moment: the neurobiological key to unlocking consciousness. Cogprints. Available online at: http://cogprints.org/3125/

Metzinger, T. (2003). Being No One. Cambridge, MA: MIT Press.

Naish, P. (2001). Hypnotic time perception: busy beaver or tardy timekeeper. Contemp. Hypn. 18, 87-99. doi: 10.1002/ch.222

Nir, Y., and Tononi, G. (2010). Dreaming and the brain: from phenomenology to neurophysiology. Trends Cogn. Sci. 14, 88-100. doi: 10.1016/j.tics.2009.12.001

Noreika, V., Valli, K. V., Lahtela, H., and Revonsuo, A. (2009). Early-night serial awakenings as a new paradigm for studies on NREM dreaming. Int. J. Psychophysiol. 74, 14-18. doi: 10.1016/j.ijpsycho.2009.06.002

Pöppel, E. (1988). Mindworks: Time and Conscious Experience. Boston, MA: Harcourt Brace Jovanovich.

Revonsuo, A. (2003). The contents of phenomenal consciousness: one relation to rule them all and in the unity bind them. Psyche 9. Available online at: http://www.theassc.org/files/assc/2568.pdf

Revonsuo, A. (2006). Inner Presence: Consciousness as a Biological Phenomenon. Cambridge: MIT Press.
Smythies, J. R. (2003). Space, time and consciousness. J. Conscious. Stud. 10, 47-56.

Varela, F. (1999). "The specious present: a neurophenomenology of time consciousness," in Naturalizing Phenomenology, eds J. Petitot, F. J. Varela, B. Pacoud, and J.-M. Roy (Stanford, CA: Stanford University Press), 266-314.

Von Kirchenheim, C., and Persinger, M. (1991). Time distortion: a comparison of hypnotic induction and progressive relaxation procedures. Int. J. Clin. Exp. Hypn. 39, 63-66. doi: $10.1080 / 00207149108409620$

Conflict of Interest Statement: The authors declare that the research was conducted in the absence of any commercial or financial relationships that could be construed as a potential conflict of interest.

Received: 18 March 2014; accepted: 15 April 2014; published online: 01 May 2014.

Citation: Fingelkurts $A A$ and Fingelkurts $A A$ (2014) Present moment, past, and future: mental kaleidoscope. Front. Psychol. 5:395. doi: 10.3389/fpsyg.2014.00395

This article was submitted to Perception Science, a section of the journal Frontiers in Psychology.

Copyright (c) 2014 Fingelkurts and Fingelkurts. This is an open-access article distributed under the terms of the Creative Commons Attribution License (CC BY). The use, distribution or reproduction in other forums is permitted, provided the original author(s) or licensor are credited and that the original publication in this journal is cited, in accordance with accepted academic practice. No use, distribution or reproduction is permitted which does not comply with these terms. 